\title{
ANALYTICAL METHODOLOGY FOR EVALUATION OF THE EFFECTS OF DIFFERENT LEACHATE TREATMENT METHODS
}

\author{
Lennart Mathiasson', Staffan Bergström ${ }^{1,2}$, William Hogland", \\ Lennart Mårtensson' ${ }^{2}$ Britt-Marie Svensson ${ }^{2}$ \\ 'Department of Analytical Chemistry, Lund University, Sweden \\ ${ }^{2}$ School of Engineering, Kristianstad University, Kristianstad, Sweden \\ ${ }^{3}$ Department of Technology, Kalmar University, Sweden
}

\begin{abstract}
Characterisation of the efficiency of leachate treatment methods when considering different groups of pollutants as phenolic compounds, PCB,s, PBDEs and phthalates is discussed. Strategies for measurements, identification and quantifications of the organic pollutants are presented and the use of supported liquid membrane extraction (SLM) and micro porous membrane extraction (MMLLE) in particular, where small and medium molecules are separated from large molecules. Acute and chronic toxicity tests by biological methods and the choice of organisms are also presented as well as the LAQUA-protocol used at the pilot plant in Kristianstad, Sweden. In acute toxicity tests of the leachate a clear accommodating on the immobility of the Artemia larvae and increasing concentration of leachate was noted. Different species react different on different compounds and therefore on complex effluents as landfill leachates the use of a battery of bioassays is recommended.
\end{abstract}

\section{KEYWORDS}

Leachate characterisation; measurements; evaluation; analytical methods; organic pollutants; treatment.

\section{INTRODUCTION}

The leachate water from a landfill has a very complex composition of salts, heavy metal ions and organic pollutants. Furthermore the content of particles in the wastewater makes a characterisation still more difficult, since different pollutants might be adsorbed onto or included in the particles. Accordingly to make conclusions whether a certain treatment procedure might lead to improvement of the water quality analytical methods for different pollutants are needed. For salt and heavy metal ions methods are established, which in most cases are expected to give sound results. However, validated methods for characterisation of organic pollutants in leachate water still need to be developed. 
The demands on analytical methods for organic pollutants differ in some respects to the demands when measuring inorganic species. In the latter case it is often the acute toxicity, which becomes a problem. For example the existence of too high concentrations of chloride ions, ammonia or heavy metal ions or $\mathrm{pH}$-related precipitation of iron from leachate water may fast lead to the death of many aquatic organisms living in this environment. However, many organic pollutants act on the endocrine systems and, since they often are bio concentrated, even very low concentrations in the leachate water may after a longer exposure period lead to severe problems for exposed organisms. This means, that analytical methods in many cases, may need to have detection limits in the medium $\mathrm{ng} / \mathrm{ml}$ range, i.e. in the ultra trace analytical region. Example of compounds which needs to be determined at very low concentrations are PCBs and polybrominated diphenyl ethers, PBDEs (flame-retardants)

Since most of the organic pollutants are unknown, a characterisation of the efficiency of a treatment method must start by considering the efficiency of the treatment with respect to different important groups of pollutants. Such groups of pollutants are phenolic compounds, PCBs, PBDEs and phthalates.

During recent years it has become generally accepted that chemical data themselves do not allow evaluating the global toxic effect that may result from the leaching out of the chemicals from the landfills. As a result, increasing attention is focussed on the incorporation of toxicity tests in hazard evaluations of waste dumps, and contact tests and bioassays are performed on leachate with aquatic organisms [1].

\section{STRATEGY FOR THE MEASUREMENTS OF ORGANIC POLLUTANTS}

\section{Group separations}

With very complex samples as leachate from landfills a good starting point for an analysis is a sample preparation step dividing the substances into groups with similar chemical properties. One starting point can then be the hydrophobicity of the molecules. Molecules with high hydrophobicity are easy to extract from water using an organic solvent or a hydrophobic solid adsorbent as $\mathrm{C}_{18}$ packing, used in solid phase cartridges or as sorbents in extraction discs (e.g. Empore discs). Interesting target substances can be released and collected using an organic solvent or supercritical carbon dioxide. In the former case the resulting solvent volume is often relatively large, which often leads to too high detection limits. Then an evaporation step will be necessary with risk for analytic losses. In the latter case the volume of the collection solvent is lower, especially if SFE equipment with a solid phase trap is used, where the collection volume may be as small as $1-1.5 \mathrm{ml}$.

For molecules, which are relatively easily dissolved in water, membrane technology can be of help for group separations. Dialysis can be used for separating small size pollutant molecules from proteins and larger humic substances. However, a more efficient way is normally to use supported liquid membrane (SLM) extraction or microporous membrane extraction (MMLLE) [2-5].

In both SLM and MMLLE the extraction unit is incorporated into a flow system. The sample stream enters the donor side of the device consisting of two blocks clamped together and with a microporous teflon membrane between. In each block are two identical channels engraved and the pores of the membrane are filled with a hydrophobic solvent. The analytes are 
transferred by diffusion from the donor side into the membrane liquid and further on to the (normally) stagnant solution in the acceptor channel at the same time as the sample passes through the device as a donor stream. Trapping of the compunds is often made by proper adjustment of $\mathrm{pH}$. For example a selective enrichment of basic compounds can be done by keeping the donor solution basic so that the basic compounds are non-protonated and can dissolve in the membrane liquid. The acceptor solution in this case is acidic leading to protonation of the basic compounds and hence an efficient trapping. By adjusting the flow rate, high extraction efficiency of the target compounds can be achieved.

The SLM methodology works very well for ionizable or permanently charged target species (including heavy metal ions). With SLM a good selectivity can be obtained and at the same time an enrichment of the target substances is achieved during the extraction. MMLLE may be used as an alternative to the solid phase extraction procedure discussed above.

By proper adjustment of the $\mathrm{pH}$ values on the donor and acceptor sides of the membrane unit water organic acids and bases can easily be separated using SLM. Neutral molecules can be extracted with MMLLE methodology.

By using methodology described above small and medium size molecules can be separated from large molecules and divided into three groups; basic, acidic and neutral compounds.

\section{Difference measurements}

Since the leachate water sample contains so many compounds, which makes identification of all these substances unrealistic, it is better to study the improvements after a treatment procedure by measuring total detector signal from groups of substances with similar properties. Naturally one must then be sure that important pollutants will be found in one of these groups. By measuring the differences obtained in the leachate samples before and after treatment the problem of having limited knowledge about recoveries of different substances will be less accentuated, provided the measurements are done as standardised as possible.

\section{Identification and quantitation of important pollutants}

The strength of the improvement accomplished by a certain treatment procedure will increase, when the fate of as many individual components as possible is known. This means that identification and determination of concentrations of components in the sample needs to be done. One way is to use final analysis steps as gas chromatography (GC) or liquid chromatography (LC), where retention times can be used for identification and peek area or peak height for quantitation. The identification safety can be further improved by using selective detectors as a diode array detector (DAD) for LC or MS connected to GC or LC.

However, one must have in mind that for each substance, which becomes identified and quantified the cost for the analysis increases, which must lead to a compromise between how many of the unknown peaks one want to identify and how many one can afford to process. In practise the best way is most probably to concentrate on certain substances, which are expected to appear and which have known toxic effect. Making the judgement one should consider as well the problem of acute toxic compounds (e.g. phenols) as those with effects after long-term exposure, often at very low concentrations, e.g. PCBs and other estrogenic compounds. 


\section{BIOLOGICAL TESTS}

The general approach for measuring toxicity is incubation of a number of organisms in a dilution series of the sample. After a defined incubation period the number of organisms suffering a defined effect (e.g. death, growth, immobility) is recorded. The percentage of organisms affected (response) is plotted against the concentrations which ideally gives a sigmoidal curve. This curve may be fitted by linear regression using probit transformed response-data and concentrations on a logarithmic scale and limits of confidence calculated. The most frequently used toxicity figure is the concentration which gives $50 \%$ response ( $\mathrm{LC}_{50}$ : concentration lethal to $50 \%$ of the organisms; $\mathrm{EC}_{50}$ : effect-concentration for $50 \%$ of the organisms) [6].

Two mayor groups of test types may be defined: acute and chronic toxicity tests. Acute toxicity tests are designed to evaluate the toxicity after a short-term exposure related to the life span of the organism (fish and crustaceans $24-96 \mathrm{~h}$, algae 4-6h and bacteria 5-30min). Common effect parameters for acute toxicity are morality (fish), immobility (crustaceans) and reduce photosynthesis (algae). In chronic toxicity tests, adverse effects are studied under conditions of long-term exposure at concentration proven to be non-lethal in acute toxicity tests. For organisms having a relatively short life-cycle (e.g. bacteria, small crustaceans) the test normally includes one to several generations of the organism. For organisms with longer life-cycles (e.g. fish) the studies cover especially life-stages and toxicity endpoints (e.g. reproduction) [6].

\section{Choice of organism}

Organisms who will be used in toxicity test of landfill leachate must have a high tolerance towards chloride and ammonia, because landfill leachate often contains chloride and ammonia in high concentrations. The concentration of chloride was in June $2000 \mathrm{ca} .1300 \mathrm{mg} / \mathrm{l}$ in the leachate from Kristianstad and ca. $4600 \mathrm{mg} / \mathrm{l}$ in the leachate from Siauliai, and the concentration of ammonia was in June $2000 \mathrm{ca} .210 \mathrm{mg} / \mathrm{l}$ respectively ca. $630 \mathrm{mg} / \mathrm{l}$. These concentrations are toxic to organisms living in fresh water and in the purpose not to hide other toxic compounds we choice to use Artemia salina, a brackish water organism.

Artemia salina lives in wild conditions in salt lakes and has a high tolerance towards chloride and the investigation of the tolerance towards ammonia made in this study shows a $24 \mathrm{~h} \mathrm{EC}_{50}$ on $1090 \mathrm{mg} / \mathrm{l}$, a concentration above that concentration common in landfill leachate. Artemia salina survive $24 \mathrm{~h}$ incubate in $\mathrm{pH}$ between 4 and 10 .

Artemia salina can be easily procured and the hatching is not complicated. No advanced equipment or big laboratory is necessary, and only small samples of water are needed for the Artemia toxicity test.

\section{THE LAQUA-PROTOCOL}

For accurate evaluation of the treatment methods it is crucial with accurate and precise analytical methods. Only then it is possible to design and construct proper treatment methods. In this project with emphasise on organic pollutants in leachate water the evaluation needs sophisticated analytical chemistry. Thus co-operation was established with the School of Engineering at Kristianstad University and the department of Analytical Chemistry at Lund 
University. Both departments have access to advanced analytical equipment and have great experience in environmental research. Leachate water from municipal solid waste landfills is a very complicated matrix and contains several hundreds of different chemical substances. In order to facilitate the evaluation process the LAQUA-protocol was established, figure 1 [7]. According to the protocol summary parameters such as for example $\mathrm{pH}$, nitrogen, ammonium and heavy metals were determined. The organic compounds were divided into two fractions and analysed with different methods according to their ability to be solved in water. The organic substances considered were phenolic substances, phthalates, PCBs and brominated flame retardants (PBDE). The toxicity of the leachate water was also tested before and after

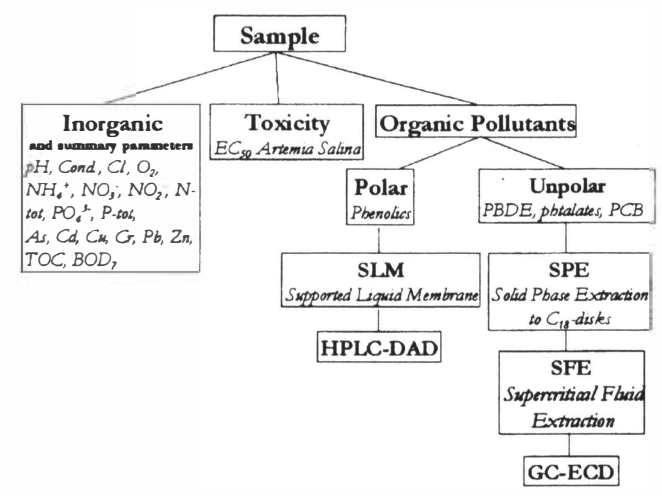

Figure 1 The LAQUA-protocol

treatment using a small crustacean (Artemia salina). The organism was exposed to the leachate water and the toxic effect was measured as number of individuals immobilised.

\section{Application of the strategy on leachate water from a landfill in Kristianstad}

In a pilot plant with leachate water obtained from the landfill in Kristianstad different treatment procedures were investigated. Water samples obtained before and after the treatment procedures were investigated. Parallel samples were extracted by SLM technique and with SPE discs, respectively.

The conditions for the SLM extraction were chosen so that phenol compounds were selectively extracted and enriched. The extraction and final analysis using LC-DAD was performed with a homebuilt-automated system. Some of the phenol compounds were identified from retention times and DAD spectra. Total peak area for a number of phenol compounds was used for estimating the efficiency of the treatment procedure.

Water samples for the SPE discs were firstly filtrated through filter paper and then through the SPE disc. The discs were then extracted with supercritical carbon dioxide in commercial, automated supercritical fluid extraction (SFE) equipment. The collected extracts were analysed by GC with electron capture detection. One ester, di-2-ethylhexyl phtalate, was identified as well as some polybrominated diphenylethers. The peak area of a number of 
substances eluting in the PCB region was used to estimate the efficiency of the treatment procedure for PCBs.

In the acute toxicity tests of leachate from Kristianstad municipal landfills a clear accommodating on the immobility of the Artemia larvae and increasing concentration of leachate was noted. In comparing the toxicity between the different treatment of leachate in the pilot plant of the LAQUA-project, the acute toxicity test with Artemia has been used. The results are an average on 4-10 tests and it is obvious that all treatment reduce the compounds how are toxic to Artemia. Less toxic is the leachate treated in the bioreactor but even the geological filters containing a mixture of peat carbon reduce the toxicity efficient. Different species react different on different compounds, and for complex effluents such as landfill leachates, the use of a battery of bioassays is recommended. The battery should be composed of test species belonging to the three trophic levels of aquatic food chains: producers, consumers and decomposers. It is it is possible to compare how different treatment of the leachate with affect the acute toxicity, but the Artemia test cannot explain what in the leachate that makes the Artemia larvae immobile. This is an acute toxicity test and you do not say anything about how the leachate will affect the organisms in the long run.

\section{REFERENCES}

1. Bernard C, G Persoone, C Janssen and A Le Du'-Delepierre (1996) Estamination of the hazard of landfills through toxicity testing of leachate, Chemosphere Vol. 33, No. 11

2. Jönsson, J. A.,Mathiasson, L., Liquid membrane extraction in analytical sample preparation. I. Principles. Trends Anal. Chem. 1999, 18, 318-325

3. Jönsson, J. Å.,Mathiasson, L., Liquid membrane extraction in analytical sample preparation. II. Applications. Trends Anal. Chem. 1999, 18, 325-334

4. Jönsson, J. A.,Mathiasson, L., Membrane extraction techniques in bioanalysis. Chromatographia Supplement 2000, 52, S8 - S11

5. Jönsson, J. A.,Mathiasson, L., Membrane-based techniques for sample enrichment J. Chromatogr. A 2000, 902, 205-225

6. Kristensen P (1992) Ecotoxicologial Characteristics of Landfill Leachate, Water Quality Institute, 11 Agem Alle', DK-2970 HØRSHOLM, Denmark.

7. Bergström, S, Mårtensson, L., and Mathiasson, L. 2000. Evaluation of the efficiency, with respect to organic pollutants, of pilot plant for local treatment of leachate water from a solid waste deposit. The $10^{\text {th }}$ symposium on handling of environmental and biological samples in chromatography. April 01-04, 2001, Mainz, Germany, p.91. 